\title{
Microstructural properties of non-supported microporous ceramic membrane top-layers obtained by the sol-gel process
}

\author{
R.S.A. de Lange, J.H.A. Hekkink, K. Keizer ${ }^{*}$, A.J. Burggraaf \\ University of Twente, Faculty of Chemical Technology, Laboratory for Inorganic Chemistry, Materials Science and Catalysis, PO Box 217 , \\ 7500 AE Enschede, The Netherlands
}

Received 19 August 1994; revised 24 August 1995

\begin{abstract}
Dried and calcined non-supported membrane top-layers of $\mathrm{SiO}_{2}, \mathrm{SiO}_{2} / \mathrm{TiO}_{2}, \mathrm{SiO}_{2} / \mathrm{ZrO}_{2}\left(10,20\right.$ and $30 \mathrm{~mol} \% \mathrm{TiO}_{2}$ and $\mathrm{ZrO}_{2}$, respectively) and $\mathrm{SiO}_{2} / \mathrm{Al}_{2} \mathrm{O}_{3}\left(10 \mathrm{~mol} \% \mathrm{AlO}_{1.5}\right)$ were prepared using acid catalyzed hydrolysis and condensation of alkoxides in ethanol. The microstructure was determined using nitrogen physisorption. The modified Horváth-Kawazoe model for nitrogen adsorption in cylindrical pores was used for pore size assessment. $\mathrm{SiO}_{2}$ non-supported membrane top layers were $100 \%$ microporous with an average porosity of 30-37\%, depending on drying conditions. The bimodal pore size distribution shows a maximum at an effective pore diameter of $0.5 \mathrm{~nm}$, and a broader tail with a weaker maximum around $0.75 \mathrm{~nm}$. Microporous non-supported binary systems can be prepared with porosities between 15 and $40 \%$. The high reactivity of the $\mathrm{Ti}, \mathrm{Zr}, \mathrm{Al}$-alkoxides requires carefully chosen conditions. Too much water results in dense materials. The pore size distributions (PSDs) of the binary systems resemble the PSDs for silica.
\end{abstract}

\section{Introduction}

Microporous ( $r_{\text {pore }}<1 \mathrm{~nm} \mathrm{[1])} \mathrm{ceramic} \mathrm{mem-}$ branes are very attractive systems due to their enhanced gas separation properties compared with mesoporous membranes. A successful method for preparing these membranes is sol-gel modification of mesoporous membranes using weakly branched polymeric sol structures, with low fractal dimensions $\left(D_{\mathrm{f}} \approx 1.5\right)[2-5]$. In a previous work, we described extensively the synthesis and characterization, by $\mathrm{SAXS}$, of polymeric $\mathrm{SiO}_{2}, \mathrm{SiO}_{2} / \mathrm{TiO}_{2}, \mathrm{SiO}_{2} / \mathrm{ZrO}_{2}$

\footnotetext{
* Corresponding author. Tel: + 3 1-53489 2998. Telefax: + 31 53433 9546. E-mail: keizer@ct.utwente.nl.
}

and $\mathrm{SiO}_{2} / \mathrm{Al}_{2} \mathrm{O}_{3}$ sols [6]. The sols are prepared by acid catalyzed hydrolysis of alkoxides in alcohol and consist of polymeric structures with fractal dimensions in the order of 1.5 or less, with Guinier radii in the order of $2-3 \mathrm{~nm}$.

In this paper, the structural properties of dried and calcined non-supported membranes prepared from these sols is discussed. The synthesis of silica and binary materials was optimized to non-supported materials which are $100 \%$ microporous, with a as possible porosity. The term non-supported materials can be somewhat confusing. In practice, a ceramic membrane consists of a support and a top-layer (or more top-layers). The support is needed for the mechanical strength of the membrane system but should have a small resistance for the gases to separate. The func- 
tional membrane (top-layer) is deposited on top of the support. Both selectivity and permeation rate should be as high as possible. Therefore, the membrane top-layer thickness should be as small as possible. Non-supported membranes consist of top-layer material only and are used for the structural characterization of the membranes. These characteristics are prerequisites for highly selective membranes, with high permeation rates. The results of this optimization process for these materials are shown. Non-supported membranes cannot be used to measure transport properties because of lack of mechanical strength.

One of the techniques used for characterization of porous materials is gas physisorption. The main goal of these experiments with non-supported microporous membrane top-layers, is to optimize and, if possible, to predict the microstructure of supported membrane top-layers. An adequate method for direct analysis of the pore structure of supported membranes is lacking, since an extremely thin top-layer, in the order of $100 \mathrm{~nm}[2,4]$ is formed. Consequently, gas physisorption analysis of supported membranes is not possible because the amount of top-layer material is much to small, and the relative influence of the mesoporous support is, therefore, strong. Permporometry, which is a relatively new technique for pore size analysis of supported membranes $[7,8]$, can not be applied for microporous systems, since the thickness of the $t$-layer [9] exceed the size of the micropores ( $t$-layer is adsorption layer of liquid used for permporometry).

Gas physisorption on microporous solids yields Type $I$ isotherms [1,9]. Since the analysis of these measurements with conventional methods, such as BET-analysis and methods based on the Kelvin equation, fail due to enhanced adsorption in micropores, a different approach for pore size assessment is necessary. Based on an evaluation of different theories and models, we selected the Horváth and Kawazoe (HK) method [10], modified according to Saito and Foley [11] for cylindrical pore geometry [12]. The HK approach is an attractive way for analysing microporous materials because of its physically plausible basis and the relatively simple calculation method, as has been shown by calibration with zeolites.

For the determination of the micropore volume, we have selected the $t$-plot method, developed by de Boer and co-workers $[13,14]$, and the DubininRadushkevich method [15], as has been described extensively elsewhere [12]. The DubininRadushkevich method is based on Eq. (1):

$V=V_{0} \exp -(A / E)^{2}$,

where $V$ is the amount adsorbed at relative pressure $P / P_{0}, V_{0}$ is the micropore volume, and $A=$ $R T \ln \left(P_{0} / P\right)$ is the adsorption potential, where $R$ is the gas constant, $T$ is the temperature $(\mathrm{K})$, and $E$ is an energy constant. The Dubinin plot is obtained by plotting $\log (V)$ as function of $\log ^{2}\left(P_{0} / P\right)$. For microporous materials, a linear part is present, in which $V_{0}$ is the intercept of this linear part at the $\log (V)$ axis.

The volume adsorbed at the plateau level of the isotherm is a measure for the total pore volume of the sample. If no mesoporosity is present, as can be analyzed with the $t$-plot, and the external volume is negligible, the plateau, therefore, gives the micropore volume, as well.

\section{Experimental}

\subsection{Non-supported membrane formation}

Polymeric $\mathrm{SiO}_{2}, \mathrm{SiO}_{2} / \mathrm{TiO}_{2}, \mathrm{SiO}_{2} / \mathrm{Al}_{2} \mathrm{O}_{3}$ and $\mathrm{SiO}_{2} / \mathrm{ZrO}_{2}$ sols were prepared by acid catalyzed hydrolysis and condensation of alkoxides in alcohol [5]. Silica sols were prepared using TEOS (tetraethyl-ortho-silicate, Merck, p.a.), ethanol (Merck, p.a.), $\mathrm{HNO}_{3}$ (Merck, p.a.) and de-mineralized water. A mixture of acid and water was carefully added to the TEOS/ethanol solution using a dropping funnel. The reaction mixture was refluxed for $3 \mathrm{~h}$ at $80^{\circ} \mathrm{C}$ under stirring. The standard sol composition $\left(\mathrm{StSiO}_{2}\right)$ is given. by the molar ratios. TEOS $/ \mathrm{H}_{2} \mathrm{O} /$ ethanol $/ \mathrm{HNO}_{3}=1 / 6.4 / 3.8 / 0.085$. The ratios, $r_{\mathrm{w}}$ and $r_{\mathrm{H}+}$, are the water/TEOS ratio and the $\mathrm{HNO}_{3} / \mathrm{TEOS}$ ratio, respectively.

The main route for the synthesis of the binary materials is a single-step prehydrolysis of TEOS followed by the addition of the $\mathrm{Ti}, \mathrm{Zr}$ or Al-alkoxide in alcohol. The alkoxides used were $\mathrm{Ti}\left(\mathrm{O}^{i} \mathrm{Pr}\right)_{4}$ (Merck, p.a. grade), $\mathrm{Ti}\left(\mathrm{O}^{n} \mathrm{Bu}\right)_{4}$ (Merck, p.a. grade), 
$\mathrm{Zr}\left(\mathrm{O}^{n} \mathrm{Bu}\right)_{4},{ }^{n} \mathrm{BuOH}$ complex (Alfa products, p.a. grade) and $\mathrm{Al}\left(\mathrm{O}^{n} \mathrm{Bu}\right)_{2}$.etac (Alfa products p.a. grade). This single-step prehydrolysis route was in some cases modified by the addition of a second amount of water and acid $\left(\mathrm{HNO}_{3}\right)$, after the addition of the composite alkoxide, in a so called two-step hydrolysis process.

The exact conditions for the binary samples are described in detail elsewhere [6]. The sample codes are identical as defined in [6] as $\operatorname{SiM} x x$, where $M$ is the binary component $(\mathrm{M}=\mathrm{Ti}, \mathrm{Zr}$ or $\mathrm{Al})$ and $x x$ is the molar percentage $\mathrm{MO}_{y}$. For the $\mathrm{SiO}_{2} / \mathrm{TiO}_{2}$ samples, the Ti-alkoxide used is given by the additional abbreviations $\mathrm{Bu}$ (Ti- $n$-butoxide) or Pr (Ti-iso-propoxide).

Non-supported microporous membranes were prepared by drying the sols in petri-dishes. Drying took place overnight under ambient conditions or during 3 $\mathrm{h}$ in a climate chamber (Hereaus Vötsch VTRK 300) at $40^{\circ} \mathrm{C}$ and $60 \%$ relative humidity and turbulent aerodynamic conditions (wind velocity $3.25 \mathrm{~m} \mathrm{~s}^{-1}$ ). No coherent layers were obtained after drying, but the xerogels were cracked in flakes of $1 \mathrm{~mm}$ to $1-2$ $\mathrm{cm}$. The mean thickness of the non-supported membranes is $1-2 \mathrm{~mm}$. Unless otherwise stated, all dried xerogel samples were calcined in static air at $400^{\circ} \mathrm{C}$ for $3 \mathrm{~h}$, with a heating and cooling rate of $25^{\circ} \mathrm{C} \mathrm{h}^{-1}$.

\subsection{Nitrogen physisorption}

Nitrogen adsorption measurements were performed volumetrically. A Carlo Erba Sorptomatic 1900 apparatus was used, extended with a turbo molecular pump system (Leybold PT50) and an extra pressure transducer (MKS Baratron type 122A) for the low pressure range $\left(10^{-3}\right.$ Torr to 10 Torr, accuracy $\pm 0.5 \%$ of reading, smallest suggested pressure reading, $3 \times 10^{-3}$ Torr). All samples were degassed for $23 \mathrm{~h}$ at $350^{\circ} \mathrm{C}$ at $10^{-6}$ Torr. Equilibrium times at low relative pressures $\left(P / P_{0}<10^{-2}\right)$ proves to be in the order of $1-2 \mathrm{~h}$ for the microporous samples (amorphous silica based materials and zeolites); at higher relative pressures, equilibrium times were between 15 and $30 \mathrm{~min}$. The degassed sample weight was used for the calculations. The experimental error in the total adsorbed volume, mainly caused by weighing errors, was found to be in the order of $5 \%$. The experiments were performed on fresh samples (within 1 day after calcination) in order to avoid aging effects.

The standard isotherm for the $t$-plot was selected according the method of Lecloux and Pirard [16], based on the calculated $C_{\mathrm{BET}}$. Porosities were calculated by

$\epsilon(\%)=\frac{V_{\mathrm{p}}}{V_{\mathrm{p}}+1 / \rho} \times 100 \%$

where $V_{\mathrm{p}}$ is the pore volume $\left(\mathrm{ml} \mathrm{g}^{-1}\right)$ and $\rho$ is the density of the solid phase $\left.(\mathrm{g} \mathrm{ml})^{-1}\right)$. A skeletal density of $2.2 \mathrm{~g} \mathrm{~cm}^{-3}$ was used [17] for amorphous calcined silica. The pore volume is calculated from the adsorbed gas volume $\left(\mathrm{ml}(\mathrm{STP}) \mathrm{g}^{-1}\right.$, at $1 \mathrm{~atm}$ and $0^{\circ} \mathrm{C}$ ) assuming ideal gas and choosing a correct estimate for the density of the adsorbed phase. For nitrogen: $V_{\mathrm{p}}\left(\mathrm{ml}\left(\right.\right.$ liq. $\left.\left.\mathrm{N}_{2}\right) \mathrm{g}^{-1}\right)=V_{\text {ads }}\left(\operatorname{ml}(\mathrm{STP}) \mathrm{g}^{-1}\right)$ $\times\left(1.547 \times 10^{-3}\right)$, where the density of nitrogen in the pores was assumed to be $0.808 \mathrm{~g} \mathrm{ml}^{-1}$, which is the density of liquid nitrogen.

In some cases, the determination of the micropore volume from the $t$-plot is not unambiguous, due to significant downward deviation from the straight line through the plateau at low $t$-values. Therefore, two values for the calculated micropore volume according to the $t$-plot method will be given, from a fit between $3<t<9$, and from the fit of the plateau region [12].

\section{Results}

\subsection{Microporous non-supported silica membrane top-layers}

\subsubsection{Influence of drying on the microstructure of standard silica samples}

$\mathrm{N}_{2}$-adsorption isotherms at $77 \mathrm{~K}$ for four silica samples (from four different synthesis batches and abbreviated as $\mathrm{AA}-\mathrm{AD}$, where $\mathrm{A}$ is for ambient) with standard composition $\left(\mathrm{StSiO}_{2}\right)$ are shown in Fig. 1. In all figures, the lines are drawn as guides for the eyes. These materials were dried overnight at ambient conditions and calcined at $400^{\circ} \mathrm{C}$ during $3 \mathrm{~h}$ $\left(25^{\circ} \mathrm{C} \mathrm{h}^{-1}\right.$ heating and cooling rate). The shape of the isotherms is typically type I. Analysis according to the $t$-plot method showed that no mesoporosity 


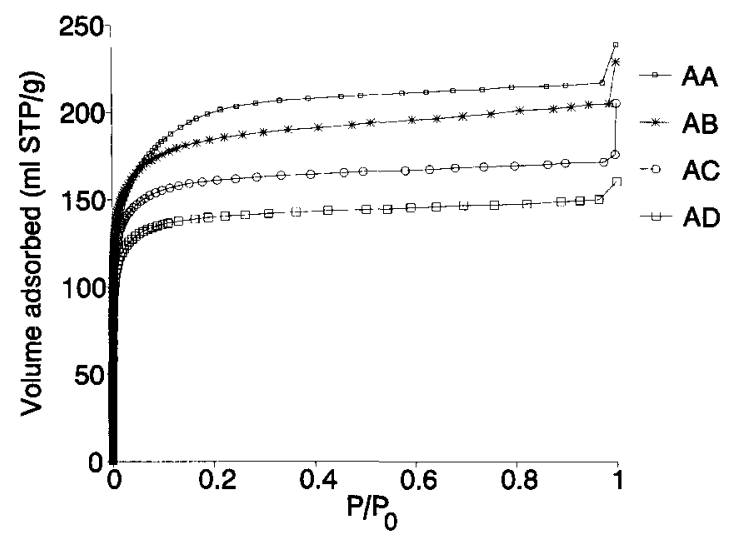

Fig. 1. $\mathrm{N}_{2}$-adsorption isotherms at $77 \mathrm{~K}$ for standard silica samples dried at ambient conditions.

was present. The micropore volume can, therefore, also be determined from the plateau-value from the isotherm. For these samples, it varies from $\approx 130 \mathrm{ml}$ $\mathrm{g}^{-1}$ to $\approx 215 \mathrm{ml} \mathrm{g}^{-1}$.

The calculated micropore volumes using the $t$-plot, isotherm intercept and according to the Dubinin method are shown in Table 1, where 'DR' points to the values obtained from the Dubinin plot and 'Isotherm intercept' to the values obtained from taken the intercept value from the isotherm. From the presented micropore volumes, it can be seen that the $t$-plot method and the isotherm intercept method give comparable micropore volumes, while the Dubinin method (DR) can give slightly smaller values. This difference is a result of the used $P / P_{0}$ range; the Dubinin method extrapolates from small $P / P_{0}$ to large $P / P_{0}$. The $t$-plot method and the isotherm intercept method extrapolate from large $P / P_{0}$ to small $P / P_{0}$.

The spread in micropore volumes of the four samples is relatively large, as could be expected from the isotherms. The calculation of the porosity results in a relatively smaller spread which, however, is still considerable.

The pore size distributions (PSDs) according to the HK model are given in Fig. 2. The plot shows that the pore size distribution of the samples is identical and consists of a maximum at $D_{\text {effective }}=0.5$ $\mathrm{nm}$ with a broader tail, with a weak maximum at $0.75 \mathrm{~nm}$, to around $D_{\text {effective }}=1 \mathrm{~nm}$. These sizes lead to the conclusion that the pore size distribution
Table 1

Micropore volumes of silica samples with standard composition using the $t$-plot, the Dubinin-Radushkevich method and the isotherm intercept method. Porosities are calculated with the 'isotherm intercept' method

\begin{tabular}{|c|c|c|c|c|}
\hline \multirow[t]{2}{*}{ Sample } & \multicolumn{3}{|c|}{$V_{\text {micro }}\left(\mathrm{cm}^{3} \mathrm{~g}^{-1}\right)$} & \multirow{2}{*}{$\begin{array}{l}\text { Porosity }^{a}(\%) \\
\text { isotherm } \\
\text { intercept }\end{array}$} \\
\hline & t-plot ${ }^{\mathrm{b}}$ & $\overline{D R}$ & $\begin{array}{l}\text { isotherm } \\
\text { intercept }\end{array}$ & \\
\hline AA & $0.30 / 0.33$ & 0.26 & 0.31 & 41 \\
\hline $\mathrm{AB}$ & $0.27 / 0.31$ & 0.27 & 0.29 & 39 \\
\hline $\mathrm{AC}$ & $0.24 / 0.25$ & 0.24 & 0.25 & 35 \\
\hline $\mathrm{AD}$ & $0.20 / 0.22$ & 0.22 & 0.21 & 32 \\
\hline $\mathrm{CA}$ & $0.25 / 0.26$ & 0.24 & 0.25 & 36 \\
\hline CB & $0.17 / 0.19$ & 0.19 & 0.17 & 27 \\
\hline $\mathrm{CC}$ & $0.18 / 0.19$ & 0.18 & 0.18 & 28 \\
\hline $\mathrm{CC}_{\mathrm{x}-\mathrm{gel}}$ & $0.34 / 0.36$ & 0.33 & 0.33 & 42 \\
\hline $\mathrm{CD}$ & $0.17 / 0.17$ & 0.18 & 0.17 & 27 \\
\hline
\end{tabular}

${ }^{\text {a }}$ Calculated with relation (2) and based on a skeletal density of amorphous silica of $2.2 \mathrm{~g} \mathrm{~cm}^{-3}$.

${ }^{b}$ Calculated from a fit in the region $3<t<9$ (first figure) and from a fit of the plateau region.

The calculated error in $V_{\text {micro }}$ is about 1 in the last figure given (example sample AA: $\mathrm{DR}=0.26 \pm 0.01$ )

The calculated error in Porosity is about 2 for values larger than $20 \%$ and 1 for values smaller than $20 \%$ both in the last figure given.

The same calculated errors hold for figures given in Tables 2-4.

can be bimodal with maxima around $0.5 \mathrm{~nm}$ and $0.75 \mathrm{~nm}$.

Silica samples CA-CD were prepared under standard conditions, but dried in a climate chamber $(3 \mathrm{~h}$

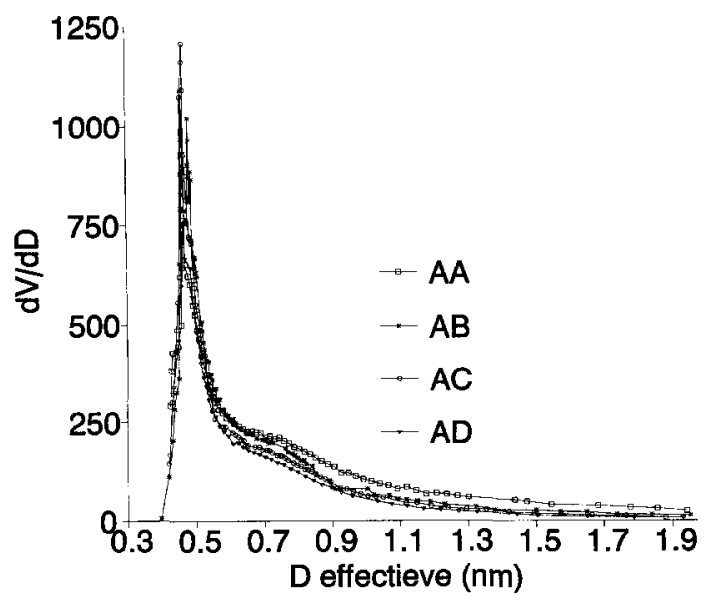

Fig. 2. Differential pore size distribution according to the HK model for silica samples dried at ambient conditions. 


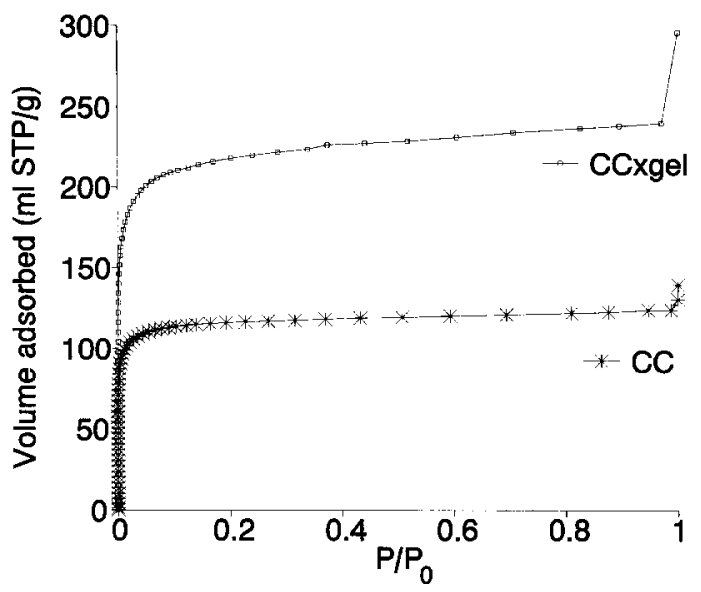

Fig. 3. $\mathrm{N}_{2}$-adsorption isotherms of a dried $\left(3 \mathrm{~h} 40^{\circ} \mathrm{C}, 60 \% \mathrm{RH}\right)$ silica xerogel $\mathrm{CC}_{\mathrm{x} \cdot \mathrm{gel}}$ and corresponding calcined sample $\mathrm{CC}$.

$\left.40^{\circ} \mathrm{C}, 60 \% \mathrm{RH}\right)$. For a typical sample $\mathrm{CC}$, the isotherm is shown in Fig. 3. Both the isotherm of the calcined silica sample $\left(3 \mathrm{~h}\right.$ at $400^{\circ} \mathrm{C}, 25^{\circ} \mathrm{C} \mathrm{h}^{-1}$ heating and cooling rate) and the isotherm of the corresponding dried xerogel $\left(\mathrm{CC}_{\mathrm{x} \text {-gel }}\right.$, degassing temperature $90^{\circ} \mathrm{C}$ ) are shown. The PSDs, according to the HK model, are given in Figs. 4 and 5. Within the experimental error, the porosities of samples, $\mathrm{CB}$, $\mathrm{CC}$ and $\mathrm{CD}$, are comparable, only sample $\mathrm{CA}$ has a considerably larger micropore volume.

Compared with the samples dried overnight under

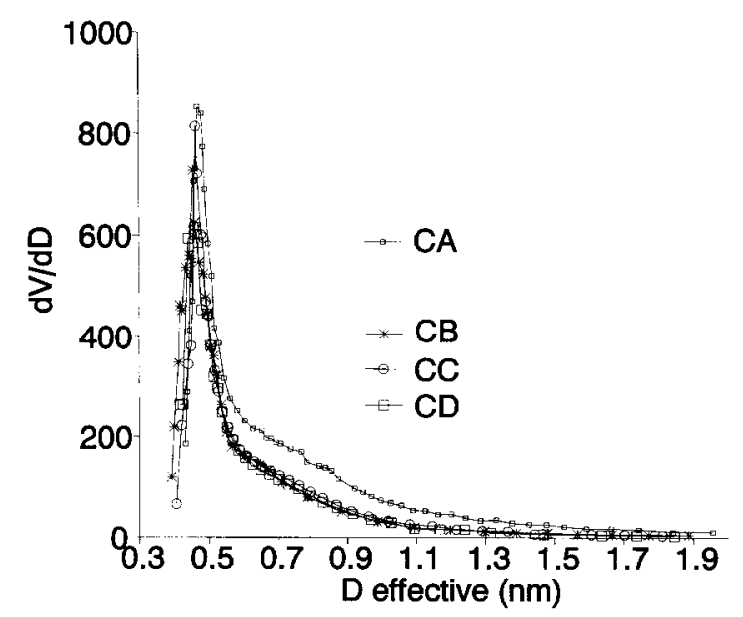

Fig. 4. Pore size distributions, according the HK model, for calcined standard silica samples (gels dried in a climate chamber for $3 \mathrm{~h}$ at $40^{\circ} \mathrm{C}, 60 \% \mathrm{RH}$ ).

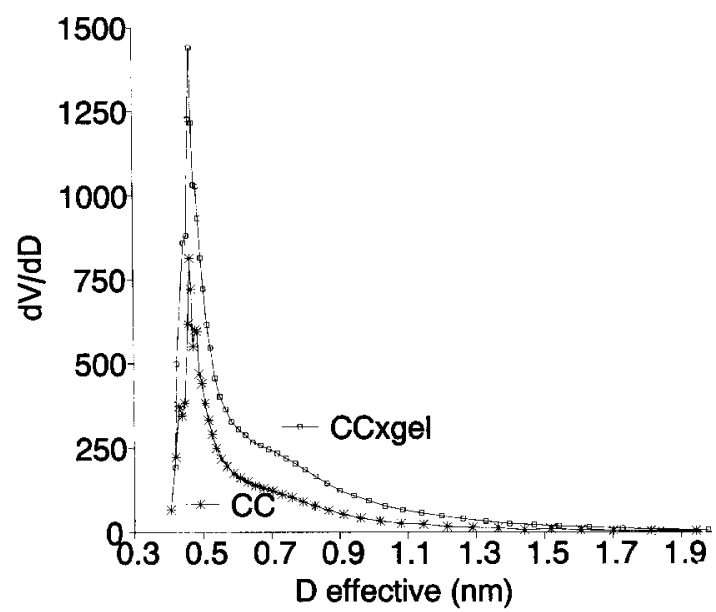

Fig. 5. Pore size distribution, according to the HK model, for dried $\left(3 \mathrm{~h}\right.$ at $\left.40^{\circ} \mathrm{C}, 60 \% \mathrm{RH}\right)$ xerogel $\mathrm{CC}_{\mathrm{x} \text {-gel }}$ and corresponding calcined sample $\mathrm{CC}$.

ambient conditions, the pore volumes are less. The higher drying rate (samples are dry in $3 \mathrm{~h}$ instead of overnight for the ambient dried samples) results in a decreased porosity.

The shapes of the calculated pore size distributions according to the HK model (Fig. 4) are surprisingly comparable with the PSDs in Fig. 2 for the samples dried overnight under ambient conditions. However, the contribution of the tail at larger pore sizes is smaller for the samples dried in the climate chamber.

The xerogel $\mathrm{CC}_{\mathrm{x} \text {-gel }}$ is $100 \%$ microporous. The porosity, however, decreases by a factor of two during calcination (sample CC). As can be seen from Fig. 5, the PSD of $\mathrm{CC}_{\mathrm{x} \text {-gel }}$ is of approximately the same shape as for the calcined material.

\subsubsection{The influence of forced drying, sol concentra- tion and sol composition on the microstructure of standard silica samples}

In the preceding sections, it has been shown that the drying rate influences the porosity of the microporous silica samples. In Table 2 , the results of $\mathrm{N}_{2}$-adsorption on microporous material dried at ambient conditions (AE, AF) are compared with forced dried samples. A forced drying process was approached by overnight drying of the sols under vacuum, obtained by a water jet pump ( $P \approx 2.4 \mathrm{kPa}=$ vapour pressure of water, sample AEJet) and a vacuum pump (Ed- 
Table 2

Micropore volumes and porosities of silica samples prepared under non-standard conditions and with non-standard compositions using the $t$-plot, the Dubinin-Radushkevich method and the isotherm intercept method

\begin{tabular}{|c|c|c|c|c|}
\hline \multirow[t]{3}{*}{ Sample } & \multicolumn{3}{|c|}{$V_{\text {micro }}\left(\mathrm{cm}^{3} \mathrm{~g}^{-1}\right)$} & \multirow{3}{*}{$\begin{array}{l}\text { Porosity }{ }^{a} \\
(\%) \\
\text { isotherm } \\
\text { intercept }\end{array}$} \\
\hline & \multirow[t]{2}{*}{$t$-plot ${ }^{b}$} & \multirow[t]{2}{*}{$\mathrm{DR}$} & \multirow{2}{*}{$\begin{array}{l}\text { isotherm } \\
\text { intercept }\end{array}$} & \\
\hline & & & & \\
\hline $\mathrm{AE}$ & $0.26 / 0.29$ & 0.26 & 0.28 & 38 \\
\hline AEjet & $0.21 / 0.22$ & 0.23 & 0.21 & 31 \\
\hline $\mathrm{AF}$ & $0.22 / 0.23$ & 0.22 & 0.20 & 31 \\
\hline AFvacuum & 0 & 0 & 0 & 0 \\
\hline $\mathrm{CA}_{\mathrm{dip}}$ & $0.11 / 0.16$ & 0.14 & 0.13 & 23 \\
\hline AG $r_{\mathrm{w}}=1$ & $0.19 / 0.21$ & 0.20 & 0.19 & 29 \\
\hline CE $r_{w}=1$ & 0 & 0 & 0 & 0 \\
\hline CF $r_{w}=12$ & $0.22 / 0.23$ & 0.22 & 0.22 & 33 \\
\hline
\end{tabular}

${ }^{a}$ Calculated with relation (2) and based on a skeletal density of amorphous silica of $2.2 \mathrm{~g} \mathrm{~cm}^{-3}$.

${ }^{b}$ Calculated from a fit in the region $3<t<9$ (first figure) and from a fit of the plateau region.

wards, $P \approx 0.1 \mathrm{~Pa}$, sample AFvacuum). After drying, the samples were calcined under static air for 3 $\mathrm{h}$ at $400^{\circ} \mathrm{C}\left(25^{\circ} \mathrm{C} \mathrm{h}^{-1}\right.$ heating and cooling rate). The pore volume of these forced dried samples is considerably smaller than for the conventional dried samples. For the sample dried with the help of the vacuum pump, no $\mathrm{N}_{2}$ adsorption was measured.

To study the effect of sol concentration on the

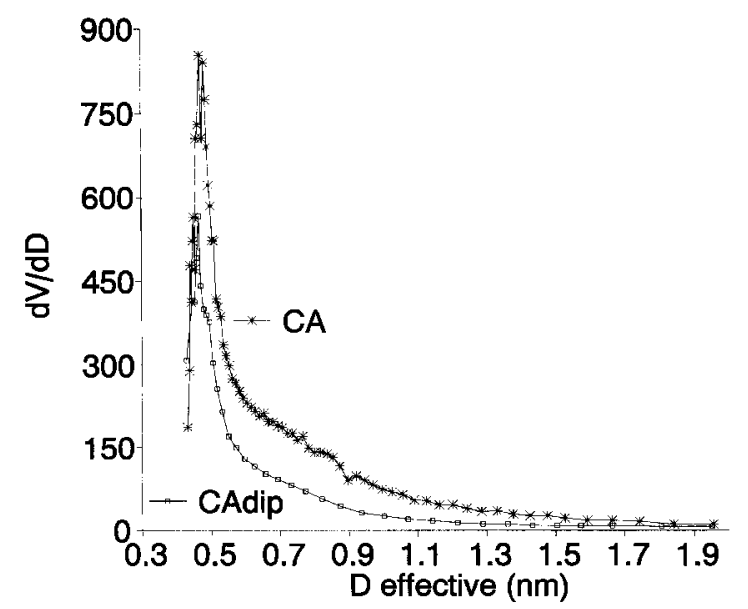

Fig. 6. Pore size distribution, according to the HK model, of silica samples $\mathrm{CA}$ and $\mathrm{CA}_{\text {dip }}$.

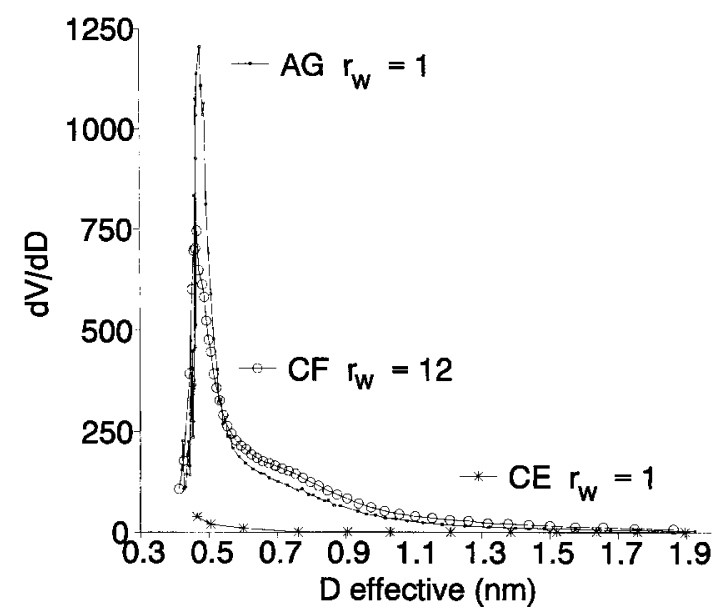

Fig. 7. Pore size distribution, according to the HK model, for calcined silica samples with non-standard $r_{\mathrm{w}}$.

microstructure development of dried silica, the $\mathrm{N}_{2}$ adsorption isotherm of sample $\mathrm{CA}$ is compared with the isotherm of the sample prepared from the same sol but diluted $18 \times$ to the level of a dip-solution $\left(\mathrm{CA}_{\mathrm{dip}}\right) . \mathrm{CA}_{\mathrm{dip}}$ is also dried in the climate chamber and calcined. Since the total volume of this diluted dip-solution was the same as for the dried sols, the layer thickness is $18 \times$ smaller and, consequently, the drying rate is higher. Both isotherms are type $\mathrm{I}$, but the porosity of $\mathrm{CA}_{\text {dip }}$ is less than measured for CA (23\% and $36 \%$, respectively). The pore size distribution of the samples is shown in Fig. 6. It can be seen that, in spite of the smaller porosity, the position of the PSD maximum and the relative contribution of the maximum and the broad tail to the PSD are not changed. A first conclusion can be that the pore size analysis of undiluted sols gives an overestimate of the porosity, but a relatively reliable PSD.

In Fig. 7, the PSDs of samples with non-standard water/TEOS ratio, $r_{\mathrm{w}}$, are given. Samples CE and CF are prepared with an $r_{\mathrm{w}}$ of 1 and 12, respectively (standard $r_{\mathrm{w}}=6.4$ ), and are dried in the climate chamber. Sample AG is prepared with $r_{\mathrm{w}}=1$ and is dried overnight at ambient conditions. All samples are calcined for $3 \mathrm{~h}$ at $400^{\circ} \mathrm{C}\left(25^{\circ} \mathrm{C} \mathrm{h}^{-1}\right.$ heating and cooling rate). The samples, $\mathrm{CF} \quad r_{\mathrm{w}}=12$ (climate chamber dried) and AG $r_{\mathrm{w}}=1$ (ambient dried), do not show different adsorption behaviour compared with the climate chamber dried samples. However, 


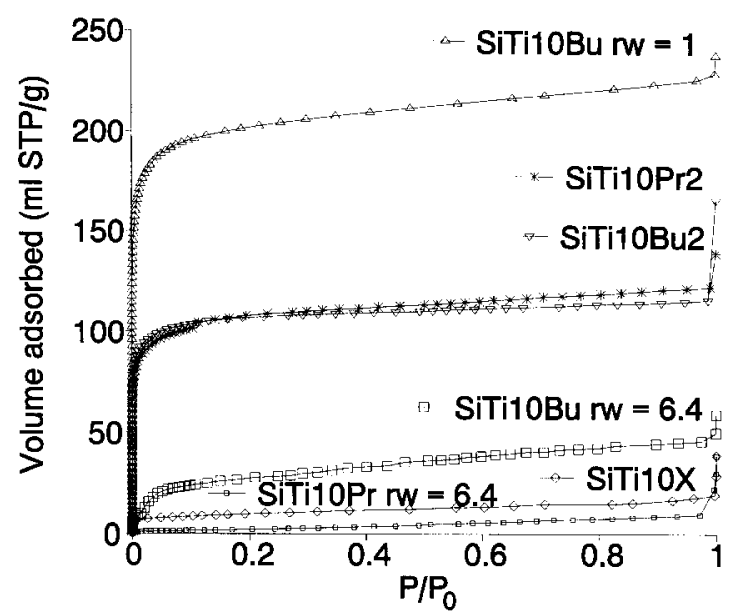

Fig. 8. $\mathrm{N}_{2}$ adsorption isotherms for $\mathrm{SiO}_{2} / \mathrm{TiO}_{2}$ (10 mol\%). SiTi10Bu2 and SiTi10Pr2, according to two-step hydrolysis, SiTiloX by mixing $\mathrm{SiO}_{2}$ and $\mathrm{TiO}_{2}$ sols.

the sample with $\mathrm{CE} r_{\mathrm{w}}=1$ (dried in the climate chamber) is dense. Obviously, the samples with small $r_{\mathrm{w}}$ values, which are only partly hydrolyzed, are more sensitive to densification at high drying rates.

\subsection{Microporous non-supported binary membrane top layers}

\subsubsection{Microporous non-supported $\mathrm{SiO}_{2} / \mathrm{TiO}_{2}$ top} layers

$\mathrm{N}_{2}$-adsorption isotherms for $\mathrm{SiO}_{2} / \mathrm{TiO}_{2}$ samples with $10 \mathrm{~mol} \% \mathrm{TiO}_{2}$ and the corresponding pore size distributions, according to the HK model, are given in Figs. 8 and 9 , respectively. The micropore volumes for the $\mathrm{SiO}_{2} / \mathrm{TiO}_{2}$ non-supported films are given in Table 3. Samples, with the general code SiTil0... $r_{\mathrm{w}}=\ldots$, are prepared using a single-step hydrolysis process and varied $r_{\mathrm{w}}$ ratio. The total amount of water and acid was added in the TEOS prehydrolysis step; $r_{\mathrm{H}+}$ was 0.085 . Samples, SiTi10Bu2 and SiTi10Pr2, are prepared using the two-step hydrolysis process with a total $r_{w}$ of 6.4 and $r_{\mathrm{H}}$ of 0.085 . The total amount of acid and water was divided for the prehydrolysis step and for the second hydrolysis step. The ratios during prehydrolysis of TEOS are $r_{\mathrm{w}}=1.14$ and $r_{\mathrm{H}+}=0.021$; the rest of the water and acid was added after the addition of the Ti-alkoxide.

Sample SiTiloX was prepared by mixing sepa-

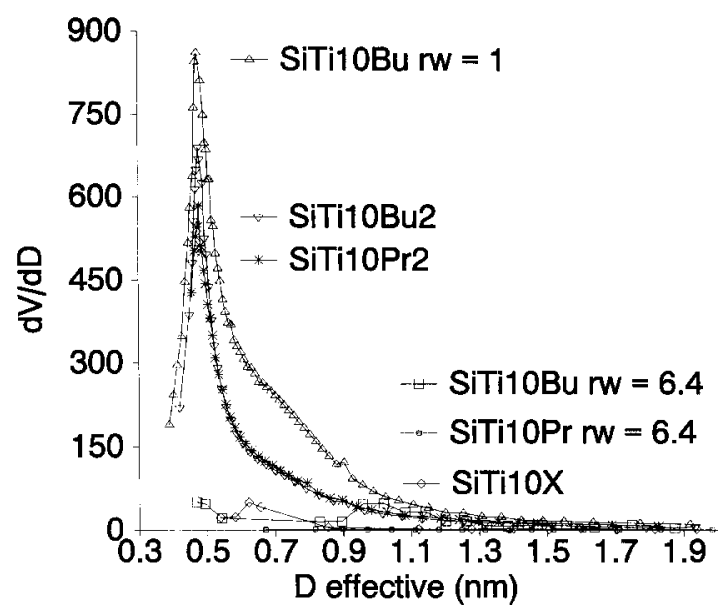

Fig. 9. PSD, according to the $\mathrm{HK}$ model, for $\mathrm{SiO}_{2} / \mathrm{TiO}_{2}(10$ mol\%) samples. SiTi10Bu2 and SiTilOPr2: two-step hydrolysis, Siti 10 by mixing $\mathrm{SiO}_{2}$ and $\mathrm{TiO}_{2}$ sols.

rately hydrolyzed $\mathrm{SiO}_{2}$ and $\mathrm{TiO}_{2}$ (using Ti-iso-propoxide) sols. All samples were dried at ambient conditions, followed by calcination for $3 \mathrm{~h}$ at $400^{\circ} \mathrm{C}$ $\left(25^{\circ} \mathrm{C} \mathrm{h}^{-1}\right.$ heating and cooling rate).

Table 3

Micropore volumes and porosites of $\mathrm{SiO}_{2} / \mathrm{TiO}_{2}$ samples using the $t$-plot, the Dubinin-Radushkevich method and the isotherm intercept method

\begin{tabular}{|c|c|c|c|c|}
\hline \multirow[t]{3}{*}{ Sample } & \multicolumn{3}{|c|}{$V_{\text {micro }}\left(\mathrm{cm}^{3} \mathrm{~g}^{-1}\right)$} & \multirow{3}{*}{$\begin{array}{l}\text { Porosity a } \\
(\%) \\
\text { isotherm } \\
\text { intercept }\end{array}$} \\
\hline & \multirow[t]{2}{*}{$t$-plot ${ }^{b}$} & \multirow[t]{2}{*}{ DR } & \multirow{2}{*}{$\begin{array}{l}\text { isotherm } \\
\text { intercept }\end{array}$} & \\
\hline & & & & \\
\hline$\overline{\text { SiTil0Bu2 }}{ }^{c}$ & $0.16 / 0.17$ & 0.17 & 0.16 & 28 \\
\hline $\operatorname{SiTi} 10 \operatorname{Pr} 2^{c}$ & $0.16 / 0.17$ & 0.17 & 0.16 & 27 \\
\hline SiTi10Bu $r_{\mathrm{w}}=6.4$ & $0.05 / 0.06$ & 0.02 & 0.03 & 7 \\
\hline SiTil0Pr $r_{w}=6.4$ & 0 & 0 & 0 & 0 \\
\hline SiTil0Bu $r_{w}=1$ & $0.32 / 0.33$ & 0.32 & 0.31 & 42 \\
\hline SiTilox & $0.02 / 0.03$ & 0 & 0.01 & 2 \\
\hline SiTi20Bu & $0.22 / 0.23$ & 0.23 & 0.21 & 35 \\
\hline $\operatorname{SiTi} 30 \operatorname{Pr} 2^{c}$ & 0 & 0 & 0 & 0 \\
\hline SiTi30Bu & $0.16 / 0.19$ & 0.16 & 0.16 & 29 \\
\hline SiTi30BuC & $0.14 / 0.14$ & 0.14 & 0.13 & 26 \\
\hline
\end{tabular}

${ }^{a}$ Calculated with relation (2) and based on a skeletal density of amorphous silica/titania of $2.36 \mathrm{~g} \mathrm{~cm}^{-3}\left(10 \mathrm{~mol} \% \mathrm{TiO}_{2}\right), 2.51 \mathrm{~g}$ $\mathrm{cm}^{-3}\left(20 \mathrm{~mol} \% \mathrm{TiO}_{2}\right)$ and $2.64 \mathrm{~g} \mathrm{~cm}^{-3}\left(30 \mathrm{~mol} \% \mathrm{TiO}_{2}\right)$.

${ }^{b}$ Calculated from a fit in the region $3<t<9$ (first figure) and from a fit of the plateau region.

${ }^{\mathrm{c}}$ Prepared according to a two-step hydrolysis process.

d Prepared by mixing of separately prehydrolyzed $\mathrm{SiO}_{2}$ and $\mathrm{TiO}_{2}$ sols. 
Fig. 8 shows that microporous materials with a high porosity can be obtained using mild conditions, which are reached by using a small $r_{w}$ in the prehydrolysis step. This condition is shown for sample SiTilOBu $r_{\mathrm{w}}=1$. The addition of a second amount of water, to $r_{w}=6.4$, in the two-step hydrolysis process for samples, SiTi10Bu2 and SiTi10Pr2, decreases the porosity. The pore size distributions, which resemble the pore size distributions of silica, show a slight decrease of the relative contribution of the tail at larger pore diameters.

A high water content in the prehydrolysis step (samples SiTil0Bu/Pr $r_{w}=6.4$ ) and the mixing of two separately hydrolyzed sols (SiTi10X) results in almost dense materials. In this paper, the term 'dense' means that there is no $\mathrm{N}_{2}$-adsorption of the membrane material at liquid $\mathrm{N}_{2}$-temperatures $(77 \mathrm{~K})$. Almost dense are materials with porosities smaller than $5 \%$.

By comparing samples, SiTi10Bu2 and SiTi10Pr2, it can be seen that, within experimental error, there is no influence of the used alkoxide. However, in the single-step process (samples, SiTil0Bu $r_{\mathrm{w}}=6.4$ and

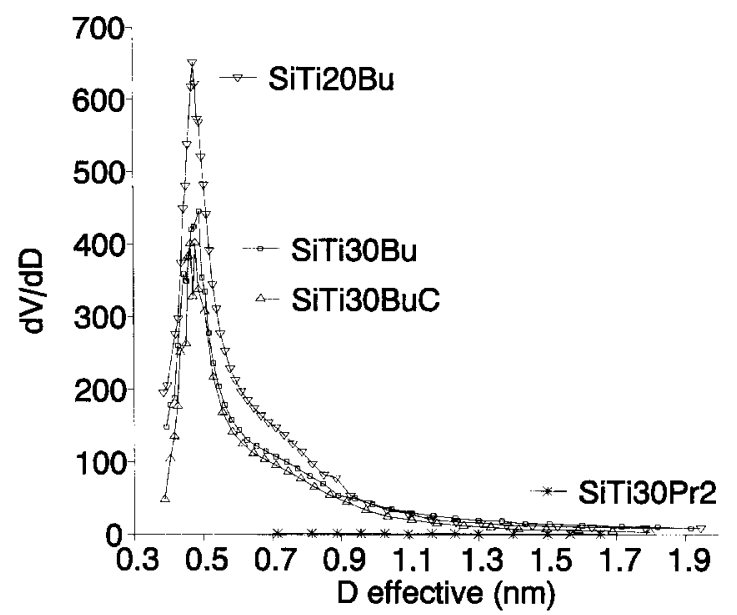

Fig. 10. PSD, according to the $\mathrm{HK}$ model, for $\mathrm{SiO}_{2} / \mathrm{TiO}_{2}(20$ and $30 \mathrm{~mol}$ ) samples. SiTi30Pr2 according to two-step hydrolysis.

SiTi10Pr $r_{\mathrm{w}}=6.4$ ), the use of Ti-isopropoxide gives a more dense system.

$\mathrm{N}_{2}$-adsorption isotherms for $\mathrm{SiO}_{2} / \mathrm{TiO}_{2}$ with 20 and $30 \mathrm{~mol} \% \mathrm{TiO}_{2}$ were typically type I. The corresponding PSDs are shown in Fig. 10. Samples

Table 4

Micropore volumes and porosites of $\mathrm{SiO}_{2} / \mathrm{ZrO}_{2}$ and $\mathrm{SiO}_{2} / \mathrm{Al}_{2} \mathrm{O}_{3}$ samples samples using the $t$-plot, the Dubinin-Rdushkevich method and the isotherm intercept method

\begin{tabular}{|c|c|c|c|c|}
\hline \multirow[t]{2}{*}{ Sample } & \multicolumn{3}{|c|}{$V_{\text {micro }}\left(\mathrm{cm}^{3} \mathrm{~g}^{-1}\right)$} & \multirow{2}{*}{$\frac{\text { Porosity }^{\mathrm{a}}(\%)}{\text { isotherm intercept }}$} \\
\hline & $t$-plot $\mathrm{b}$ & $\mathrm{DR}$ & isotherm intercept & \\
\hline SiZr 10 & $0.08 / 0.09$ & 0.08 & 0.08 & 16 \\
\hline $\mathrm{SiZr} 1 \mathrm{ON}$ & $0.12 / 0.14$ & 0.13 & 0.13 & 23 \\
\hline $\operatorname{SiZr} 10-2 r_{w}=2$ & $0.006^{c}$ & 0 & 0.008 & 2 \\
\hline SiZr $10-80$ & $0.006^{c}$ & 0 & 0.006 & 1 \\
\hline SiZr20.1 & $0.11 / 0.13$ & 0.12 & 0.12 & 27 \\
\hline SiZr20.2 & $0.13 / 0.17$ & 0.14 & 0.13 & 29 \\
\hline SiZr30.1 & $0.09 / 0.11$ & 0.10 & 0.10 & 27 \\
\hline SiZr30.2C & 0.08 & 0.07 & 0.08 & 22 \\
\hline $\mathrm{SiZr} 30.3 \mathrm{C}$ & 0 & 0 & 0 & 0 \\
\hline SiAl10 $r_{w}=4.6,70$ & $0.005^{c}$ & - & 0.004 & 1 \\
\hline SiAllo $r_{w}=2.1,20$ & $0.003 / 0.012$ & 0.009 & 0.009 & 2 \\
\hline SiAl $10 r_{w}=1.8,80$ & $0.003^{c}$ & - & 0.005 & 1 \\
\hline SiAllo $r_{w}=1.8,20$ & $0.09 / 0.11$ & 0.08 & 0.09 & 16 \\
\hline SiAllo $r_{w}=1.8,80$ & 0 & - & 0 & - \\
\hline
\end{tabular}


SiTi20Bu and SiTi30Bu are prepared using a singlestep prehydrolysis process; the total amount of water and acid was added in the TEOS-prehydrolysis step. The mol ratios, $r_{\mathrm{w}}$ and $r_{\mathrm{H}+}$, are 1 and 0.085, respectively. Sample SiTi30BuC was prepared using the same conditions, but the gel was dried in the climate chamber $\left(40^{\circ} \mathrm{C}, 60 \% \mathrm{RH}\right)$. As has been observed for the silica experiments, this drying results in a slightly smaller porosity.

Sample SiTi30Pr2 was prepared using a two-step hydrolysis process with $r_{\mathrm{w}}=1.14$ and $r_{\mathrm{H}+}=0.021$ in the TEOS prehydrolysis step and $r_{\mathrm{w}}=6.4$ and $r_{\mathrm{w}}=0.085$ as total mol ratios. The influence of this second hydrolysis step, however, as shown by comparison with SiTi30Bu (this comparison is allowed since it is shown (Table 3) that, for low $r_{\mathrm{w}}$ in the prehydrolysis step, no influence of the used alkoxide was found), is a large effect; the porosity disappears completely.

Generally, we conclude that microporous $\mathrm{SiO}_{2} / \mathrm{TiO}_{2}$ can be prepared when mild reaction conditions are used, the porosities being slightly less than for silica. The pore sizes and PSDs for these samples are roughly the same compared with silica, but a slight decrease of the contribution of the tail at wider pores is present for the samples SiTi10Bu/Pr $r_{\mathrm{w}}=6.4$.

More water leads to lower porosities or even dense materials. An important difference between samples with $10 \mathrm{~mol}_{\%} \mathrm{TiO}_{2}$ and $30 \mathrm{~mol} \% \mathrm{TiO}_{2}$ is that the two-step hydrolysis process leads to a dense material for the latter samples, while for material with $10 \mathrm{~mol} \% \mathrm{TiO}_{2}$ porosities in the order of $28 \%$ have been measured.

\subsubsection{Microporous non-supported $\mathrm{SiO}_{2} / \mathrm{ZrO}_{2}$ and} $\mathrm{SiO}_{2} / \mathrm{Al}_{2} \mathrm{O}_{3}$ top layers

Results from $\mathrm{N}_{2}$-adsorption measurements for $\mathrm{SiO}_{2} / \mathrm{ZrO}_{2}$ with 10,20 and $30 \mathrm{~mol} \% \mathrm{ZrO}_{2}$ and $\mathrm{SiO}_{2} / \mathrm{Al}_{2} \mathrm{O}_{3}\left(10 \mathrm{~mol} \% \mathrm{AlO}_{1.5}\right)$ are given in Table 4. SiZr10 is prepared using the standard single-step prehydrolysis route, with $r_{\mathrm{w}}=1$ and $r_{\mathrm{H}+}=0.085$ in the TEOS prehydrolysis step. Sample SiZr 10-2 $r_{\mathrm{w}}=$ 2 is prepared using a two-step process, with $r_{w}=1$ and $r_{\mathrm{H}+}=0.085$ (standard) in the TEOS prehydrolysis step. After the addition of the $\mathrm{Zr}$-alkoxide, a second amount of water and acid was added resulting in total ratios $r_{\mathrm{w}}=2$ and $r_{\mathrm{w}}=0.17$. An in-

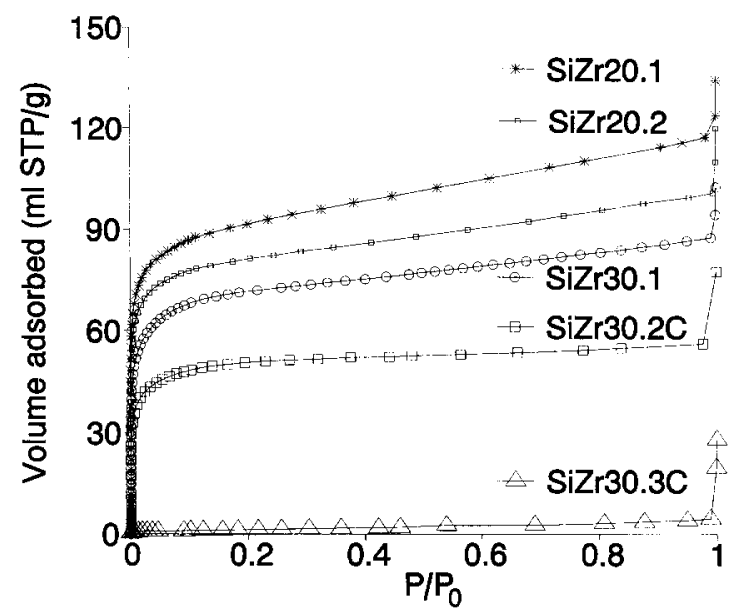

Fig. 11. $\mathrm{N}_{2}$ adsorption isotherms for $\mathrm{SiO}_{2} / \mathrm{ZrO}_{2}(20$ and 30 mol\%) samples. See text for exact synthesis conditions.

creased prehydrolysis temperature $\left(80^{\circ} \mathrm{C}\right.$ against $20^{\circ} \mathrm{C}$ for the standard process) is used for sample SiZr1080. During the TEOS prehydrolysis of sample SiZr 10N, $\mathrm{N}_{2}$ was flushed over the sol. This flushing results in evaporation of ethanol leading to a sol volume decrease of around $20 \%$.

The two-step hydrolysis process with $r_{w}=2$ results in a large decrease of the pore volume. Also increasing the TEOS prehydrolysis temperature results in a pore volume decrease. Surprisingly, the effect of alcohol evaporation (sample SiZr10N), which results in a higher concentration of the reactants, was a pore volume increase. The pore size distribution for $\mathrm{SiZr} 10 \mathrm{~N}$ is similar to $\mathrm{SiO}_{2}$ and $\mathrm{SiO}_{2} / \mathrm{TiO}_{2}$.

$\mathrm{N}_{2}$-adsorption isotherms for $\mathrm{SiO}_{2} / \mathrm{ZrO}_{2}$ samples with 20 and $30 \mathrm{~mol} \% \mathrm{ZrO}_{2}$ are given in Fig. 11. Samples, SiZr20.1, SiZr20.2 and SiZr30.1, are prepared using the same $\mathrm{Zr}$-alkoxide batch with standard $r_{\mathrm{w}}=1$ and $r_{\mathrm{H}+}=0.085$. Sample SiZr30.2C was prepared using this same batch but 1 year after opening (during which the closed bottle was kept under nitrogen). Sample SiZr30.3C was prepared using identical conditions, but with a fresh $\mathrm{Zr}$-alkoxide batch. SiZr30.2C and SiZr30.3C were dried in the climate chamber $\left(40^{\circ} \mathrm{C}, 60 \% \mathrm{RH}\right)$. The corresponding pore size distributions are shown in Fig. 12.

Compared with $\mathrm{SiO}_{2} / \mathrm{TiO}_{2}(10 \mathrm{~mol} \%)$ binary materials, the porosities of $\mathrm{SiO}_{2} / \mathrm{ZrO}_{2}(10 \mathrm{~mol} \%)$ 


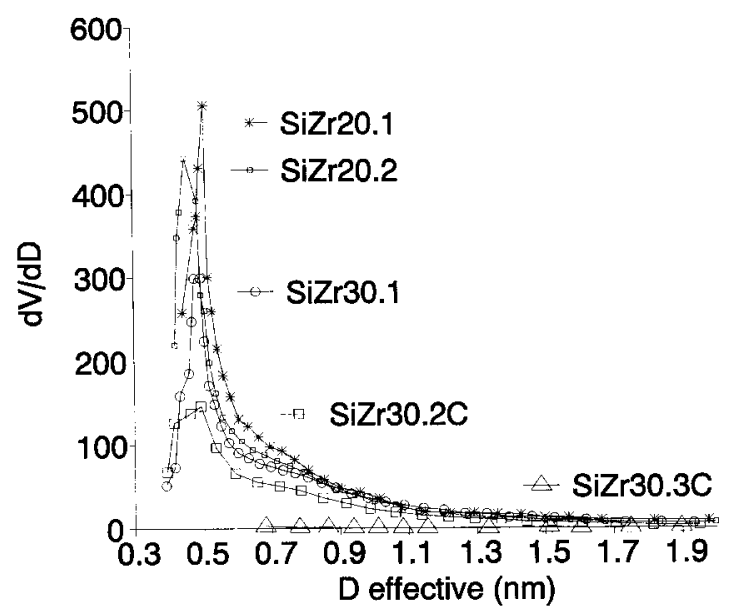

Fig. 12. PSD, according to the $\mathrm{HK}$ model, for $\mathrm{SiO}_{2} / \mathrm{ZrO}_{2}(20$ and $30 \mathrm{~mol} \%$ ). See text for exact synthesis conditions.

with comparable synthesis conditions are smaller (compare, e.g., sample SiZr10 and sample SiTi10Bu $r_{\mathrm{w}}=1$ ). The two-step hydrolysis process also shows this trend, a porosity of only $2 \%$ is found (sample SiZr10-2 $r_{\mathrm{w}}=2$ ). The PSD of highly porous sample SiZr10N is comparable with silica. Contrarily, porosities and PSDs of samples containing $20 \mathrm{~mol} \%$ and $30 \mathrm{~mol} \% \mathrm{ZrO}_{2}$ are comparable with $\mathrm{SiO}_{2} / \mathrm{TiO}_{2}$.

The effect of the drying conditions is partly in agreement with the results found for silica. As can be seen by comparison of SiZr30.1 and SiZr30.2C, fast drying in the climate chamber results in a slightly smaller pore volume. The resulting PSD after fast drying is broader, with a relatively larger contribution at wider pore diameters.

The effect of the alkoxide batch is striking; obviously, the alkoxide quality is extremely important, since the same batch gives comparable results even after 1 year, while a new batch results in dense materials (compare SiZr30.2C and SiZr30.3C).

The $\mathrm{SiO}_{2} / \mathrm{Al}_{2} \mathrm{O}_{3}\left(10 \mathrm{~mol} \% \mathrm{AlO}_{! .5}\right)$ sample code is described by the $r_{\mathrm{w}}$ ratio $(X)$ and the TEOS prehydrolysis temperature $(x \times)$, given by the general code: SiAllo $r_{\mathrm{w}}=\times, \times \times$. It can be seen that a microporous material can be obtained only if very mild conditions are used; a low prehydrolysis temperature and low water content $\left(r_{\mathrm{w}}=1.8\right)$ in the prehydrolysis step.

\section{Discussion}

\subsection{Microporous non-supported silica membrane top layers}

Ambient dried and calcined silica non-supported membrane top-layers had type I $\mathrm{N}_{2}$-adsorption isotherms with shape and micropore volumes in agreement with data in the literature [18-20]. From earlier work [6], it is known that the standard silica sols consist of weakly branched polymeric structures with a fractal dimension around 1.4 and a Guinier radius of around $2 \mathrm{~nm}$. From the theory presented, it was expected that microporous materials would result.

The non-supported membrane samples are $100 \%$ microporous, as has been shown with the $t$-plot analysis. The calculated micropore volumes using $t$-plot analysis, the DR analysis and by the simple intercept methods are comparable. The porosity range is from 32 to $42 \%$. This is a relatively wide range, which may be caused by fluctuations in atmospheric drying conditions. It is, therefore, quite remarkable that the pore size distribution of these different samples, given by the modified HK analysis, is similar. The pore size distribution for these samples is bimodal and shows a maximum at an effective pore diameter of around $0.5 \mathrm{~nm}$, and a weaker maximum at an effective diameter of around $0.75 \mathrm{~nm}$.

The effect of drying rate can be seen by comparison of the samples dried under ambient conditions and in a climate chamber. The drying times are around $16-20 \mathrm{~h}$ and $3 \mathrm{~h}$, respectively. The porosities for the samples dried in the climate chamber are in the range of $27-36 \%$, which is smaller than for the samples dried under ambient conditions, and apart from sample CA the spread in results is less. The pore size distributions are of the same shape as calculated for the ambient-dried samples, in which the relative contribution of the tail at larger pore sizes is slightly less. This effect causes a smaller pore size distribution. Forced drying under vacuum reduced the pore volume considerably too, especially in the case of the high vacuum experiment in which a dense resulted material. In this case, the PSD shows a reduced contribution of the tail at wider pore sizes. 
A possible explanation for the higher porosity of the ambient dried samples in comparison with the climate chamber dried samples and the forced dried samples is that, during the long drying process, stiffening of the gel has taken place due to condensation reactions between neighbouring polymeric clusters. Collapse of the gel structure, due to the high capillary pressure, is hindered, producing a higher porosity.

Another effect may be that growth of the polymer has taken place, resulting in a larger radius, $r$, and larger fractal dimension, $D_{\mathrm{f}}$ As has been discussed before, interpenetration of the polymers takes place during consolidation $[6,21]$. It has been shown that the dependency of density and tendency to interpenetrate on fractal dimension, and Guinier radius can be described using [22]

(1) The scaling of density of a mass fractal object:

density $\sim$ mass $/$ volume $\sim \frac{r^{D_{f}}}{r^{d}}=r^{D_{\mathrm{f}}-3}$,

where $d$ is the dimension of space and $D_{\mathrm{f}}$ is the mass fractal dimension $\left(1<D_{\mathrm{f}}<3\right)$. The density of a cluster decreases with increasing $r$ and increases with increasing $D_{\mathrm{f}}$.

(2) The tendency of fractal systems to interpenetrate, which is inversely related to the average number of intersections, $M_{1,2}$, of two mass-fractal objects of size, $r$, and mass fractal dimension placed in the same region of space:

$M_{1,2} \propto r^{2 D_{i}-d}=r^{2 D_{i}-3}$.

If $D_{\mathrm{f}}$ remains less than 1.5 during the drying process, then increase of cluster size, $r$, will result in decreasing the number of intersections, $M_{1.2}$, thus increasing the tendency to interpenetrate, which is an opposite effect compared with the higher degree of condensation during the process. It is, therefore, difficult to predict the cumulative effect of these contributions on the final porosity. However, when $D_{\mathrm{f}}$ becomes greater than 1.5 , the number of intersections will increase for higher $r$ values, leading to a decreased tendency to interpenetrate. Consequently, this trend will lead to a higher porosity. Since it is not known if $D_{\mathrm{f}}$ increases above 1.5 during the consolidation process, this is an other unknown parameter for the prediction of the final microstructure.

The sample prepared from a dip solution (Fig. 6) also showed a decreased micropore volume. This decrease can be explained by the fact that condensation reactions are almost quenched due to the high level of dilution. The resulting non-supported layer is very thin therefore, from the stage at which the same level of dilution is reached as compared with the normal sols, drying is much faster. The condensation time is, therefore, shorter.

Since the layer is thinner, however, one would also expect a stronger influence of the 'support' (petri-dish). The constraint effect of the substrate will decrease the tendency to shrink during drying, due to tensile stresses [23]. The fact that this shrinkage is not observed might be an indication that the effect of the absence of gel stiffening due to condensation is more important than the effect of the tensile stress caused by support constraints. Since the effect of tensile stress is, in fact, present for all samples, it is possible that the difference in tensile stress state is small.

The effect of the water/TEOS ratio, $r_{w}$, (Table 2, Fig. 7) shows that an increase of $r_{\mathrm{w}}$ to 12 (standard: $r_{\mathrm{w}}=6.4$ ) for a climate chamber dried sample has no significant influence on pore volume and pore size distribution. This absence is in agreement with the results from SAXS experiments in which it was found that the sol structure is comparable $\left(D_{\mathrm{f}} \approx 1.5\right.$, $R_{\mathrm{g}} \approx 20 \AA$ ) with the sol structure of silica prepared with the standard composition.

If $r_{\mathrm{w}}=1$, however, there is also no significant influence for the sample dried under ambient conditions. This small influence is remarkable, since the sols were found to consist of structures too small to produce measurable scattering with SAXS. Drying in the climate chamber, however, resulted in a dense material. Since both $D_{\mathrm{f}}$ and $R_{\mathrm{g}}$ are smaller than for silica sols prepared using the standard composition, and the fact that this sol is less reactive $\left(r_{\mathrm{w}}=1\right)$, it could be expected that consolidation results in a material with smaller porosity.

Based on the results in this section, we can expect smaller porosities and possibly smaller pores, as well, for the supported films compared with the measured porosities for non-supported films. Since the layer thickness of the film is about $100 \mathrm{~nm}$ [4] and the thickness of the non-supported systems is around $1 \mathrm{~mm}$, it is clear that the non-supported films will have more bulk-like properties. 


\subsection{Microporous non-supported binary membrane top layers}

When the adsorption results for SiTi10 are compared with the sol structure, as reported before [6], some important points arise.

- Single-step hydrolyzed sols with $r_{\mathrm{w}}=1$ were found to consist of polymeric structures which are too small to result in measurable scattering with SAXS. These sols had, in non-supported materials, a porosity of $42 \%$. (A comparable result was found for the 20 and $30 \mathrm{~mol} \% \mathrm{TiO}_{2}$ systems.)

- A larger $r_{\mathrm{w}}$ of 6.4 in the single-step prehydrolysis process leads to gels which result in nearly dense structures after calcination; these gels, however, consist of polymeric structures with a fractal dimension of $1.9 \pm 0.1$ and a Guinier radius of $5.1 \pm 0.3 \mathrm{~nm}$.

- A two-step hydrolysis process, with a total $r_{\mathrm{w}}$ of 6.4 , produces microporous materials with a moderate porosity of around $28 \%$. The fractal dimension of the sols was 1.3 and the Guinier radius was around $1.7 \pm 0.1 \mathrm{~nm}$. It was found [5] that microporous supported membranes could be successfully prepared with these sols. From permeation experiments, an activation energy for hydrogen permeation of $13 \pm 2 \mathrm{~kJ} \mathrm{~mol}^{-1}$ was measured, which indicates that molecular sieve properties have been achieved [24].

- The sol prepared by mixing two separately hydrolyzed sols results in a nearly dense material. The fractal dimension of this system was $1.95 \pm 0.10$ with a Guinier radius of $3.6 \pm 0.2$. Also with this sol, microporous membranes can be made, as has been reported elsewhere [5]. Separation factors for $\mathrm{H}_{2} / \mathrm{CH}_{4}$ exceeding 200 have been measured. In the two-step process for SiTilo no difference in the microstructure was found between sols prepared from Ti-isopropoxide and Ti- $n$-butoxide. In the single-step process with the same $r_{\mathrm{w}}$ (samples SiTil0Bu/Pr $r_{\mathrm{w}}=6.4$ ), however, the sol with Tiisopropoxide produced a denser structure compared with a sol prepared with the less reactive Ti- $n$-butoxide. In the single-step process the Ti-alkoxides can react directly with water while in the two-step process this reaction is not possible since the amount of water is substoichiometric in the prehydrolysis step. From these results, it seems that the type of titanium-alkoxide, depending on the experimental conditions, can have an influence on the microstructure.

Moreover, an increased $\mathrm{TiO}_{2}$ concentration leads to a slight decrease in porosity. This decrease can be seen by comparison of samples SiTi10Bu $r_{w}=1$, SiTi20Bu and SiTi30Bu, which show a porosity of $42 \%, 35 \%$ and $29 \%$, respectively. These samples were prepared using a single-step prehydrolosis process with $r_{\mathrm{w}}=1$. This $r_{\mathrm{w}}$ is based on the amount of $\mathrm{Si}$. Consequently, the ratio $\mathrm{H}_{2} \mathrm{O} /(\mathrm{Si}+\mathrm{Ti})$ decreases for an increased amount of $\mathrm{TiO}_{2}$.

An important difference in the sensitivity of SiTi10 and SiTi30 to the amount of water can be seen for the two-step process. For the samples, SiTi10Bu2 and SiTi10Pr2, prepared according to the two-step process, the pore volume was decreased compared with single-step process (SiTi10Bu $r_{\mathrm{w}}=$ 1 ), but still a resulting porosity of $28 \%$ was obtained. The two-step process for SiTi30Pr2, however, leads to a dense system.

For $\mathrm{SiO}_{2} / \mathrm{ZrO}_{2}$ it was found that the standard sol SiZr10 (Table 4) produced a microporous material with a moderate porosity. With SAXS, it was found that these sols consist of structures too small to be resolved. The addition of extra water results in an increase of the polymer size with a fractal dimension of $1.4 \pm 0.1$ and a Guinier radius of $0.84 \pm 0.05 \mathrm{~nm}$. The porosity, however, disappears as can be seen from sample $\mathrm{SiZr} 10-2 \quad r_{\mathrm{w}}=2$. The $\mathrm{SiO}_{2} / \mathrm{ZrO}_{2}$ sols with $30 \mathrm{~mol} \% \mathrm{ZrO}_{2}$, resulting in microporous materials with around $25 \%$ porosity, were also found to consist of such small polymeric structures.

Contrary to $\mathrm{SiO}_{2} / \mathrm{TiO}_{2}$, the porosity of $\mathrm{SiO}_{2} / \mathrm{ZrO}_{2}$ with $10 \mathrm{~mol} \% \mathrm{ZrO}_{2}$ is less than for samples prepared under the same conditions with 20 $\mathrm{mol} \% \mathrm{ZrO}_{2}$ and $30 \mathrm{~mol} \% \mathrm{ZrO}_{2}$. This difference can be seen by comparison of samples SiZr10, SiZr20.1, SiZr20.2 and SiZr30.1.

As for $\mathrm{SiO}_{2} / \mathrm{TiO}_{2}$ and $\mathrm{SiO}_{2} / \mathrm{ZrO}_{2}$, very mild conditions must be used for the synthesis of microporous $\mathrm{SiO}_{2} / \mathrm{Al}_{2} \mathrm{O}_{3}$. Sols SiA110 $r_{\mathrm{w}}=1.8,80$ and SiA110 $r_{\mathrm{w}}=2.1$, 20, which result in dense materials, were found to consist of polymeric structures too small to be resolved with SAXS. Sample SiA110 $r_{\mathrm{w}}=1.8,20$, which gave a microporous material, should, given the milder reaction conditions, consist of even smaller structures. 
Sample SiA110 $r_{\mathrm{w}}=4.6,70$, with a porosity of around $1 \%$, however, proved to be an effective microporous supported membrane [5]. From $\mathrm{H}_{2}$ permeation experiments an activation energy of $9 \pm 2 \mathrm{~kJ}$ $\mathrm{mol}^{-1}$ was calculated; this indicates that molecular sieve properties have been obtained [24]. The permeation of this membrane was considerably less (factor 10) than for standard silica membranes, which probably means that the porosity of the supported material must be less than for $\mathrm{SiO}_{2}$, as well, assuming comparable membrane thickness and mean pore size.

It is remarkable that, for the binary systems, microporous materials can be obtained from sols consisting of polymers with structures too small to be resolved with SAXS. Addition of extra water leads us, in general, to the formation of sols with larger fractal structures. Surprisingly, the consolidated systems will become more dense. This result is in contradiction with the theory of pore structure tailoring using the fractal concept, as presented before [6]. From this theory, more open structures, with higher porosity must be expected if the fractal dimension and the Guinier radius are increased.

Provided free water is present, the incorporation of the reactive alkoxides leads to strong internal condensation in the separate polymers, leaving no porosity in the internal structure of these polymers. Interpenetration of separate polymers is, therefore, difficult. The porosity of the final structure consisting of a packing of these dense clusters is loose; in some cases, however, a small amount of mesoporosity (as has been found with $t$-plot analysis) is present.

If small clusters with a very low fractal dimension are present, which is the case for binary sols with low $r_{w}$, interpenetration will take place. The reactivity of these sols is less than for binary sols with high $r_{w}$, but greater than for silica. It can be imagined that condensation takes place only when the separate structures start to interpenetrate. Further interpenetration is then hindered, which can result in a relatively open structure. Also a considerable quantity of organic groups is present, since an under-stoichiometric $r_{\mathrm{w}}$ is used. During calcination, these groups will be removed, leaving additional porosity. The total weight losses for $\mathrm{SiO}_{2} / \mathrm{TiO}_{2}$ and $\mathrm{SiO}_{2} / \mathrm{ZrO}_{2}$ are $35 \%$ and $33 \%$, respectively, which is considerably greater than for silica (23\%) [25].
The PSDs, according to the HK model for the binary systems, show a strong resemblance, both in shape of the distribution and in pore size, to the PSDs for silica. This similarity is remarkable, since the equilibrium pressures are sensitive to small changes in pore size in this pore size region [12]. In some cases, the influence of wider pores may become more important, as is the case for $\mathrm{SiO}_{2} / \mathrm{ZrO}_{2}$ and $\mathrm{SiO}_{2} / \mathrm{Al}_{2} \mathrm{O}_{3}$.

Based on these observations, comparable gas transport properties for membranes may be expected, unless the contribution of the wider pores becomes significant. This may be of importance for gas separation, as discussed elsewhere [26].

\subsection{Concluding remarks concerning microporous membrane formation}

Kumar et al. [23] found that the differences in supported and non-supported microstructure for $\mathrm{TiO}_{2}$ membranes can be considerable. Due to support constraints, the driving force for sintering is decreased and particle rearrangement is prevented. This decrease results in an increased microstructural stability, which is shown by a higher transformation temperature and a lower sinter reactivity. Differences in structural evolution of non-supported membranes with different thickness have been found too this effect, however, was small compared with the difference for supported and non-supported membranes. It is concluded that evaluation of textural properties of non-supported membranes in order to understand the properties of supported membranes is a pessimistic approach, since an underestimate of the stability of these membranes would be found.

For silica films prepared from polymeric sols, considerable differences in microstructure of the dried materials can be expected between non-supported gels and supported films. From experiments with a so-called Surface Acoustic Wave (SAW) device, it has been shown by Brinker, Frye et al. [21,27] that a supported $\mathrm{SiO}_{2}$ film prepared on the SAW device showed no $\mathrm{N}_{2}$-adsorption, while the xerogels prepared from the same sols are microporous. Initially, this result seems contrary to the differences between supported and non-supported $\mathrm{TiO}_{2}$ systems, as mentioned above. The origin for this difference, however, can be found in the timescale in which the 
consolidation process takes place. As mentioned before, the consolidation process can be described according to fractal concepts. However, condensation reactions can still take place. The ratio of condensation rate and evaporation rate is, therefore, an important parameter.

In the case of non-supported layers the drying process can take several hours, contrary to the supported systems in which drying can take place within seconds. During drying, alcohol is preferentially removed [28], leaving a xerogel with a pore fluid consisting of water and $\mathrm{HNO}_{3}$. This effect results in a less diluted system, which increases the reactivity, since concentrations are increased. However, due to the low $\mathrm{pH}$, the condensation rates are small, as shown by Coltrain et al. [29]. The mobility of the polymeric molecules is also decreased if consolidation takes place.

The increased drying time results in a more condensed structure. Collapse of the gel structure during the drying process due to the capillary forces is then difficult, and the structure remains porous. In the very fast drying process for the supported systems, which may be due to the thin layer and/or the removal of solvent in the case of a porous support, condensation can hardly take place and the polymers will interpenetrate (into each other), resulting in a nearly dense microstructure. In the case of a porous support, penetration into the pores can not be excluded if the relative pore size, with respect to the cluster size of the polymers, is large enough.

According to this model description for consolidation, it is clear that the drying conditions of the non-supported gels have to be taken into consideration when the obtained results for non-supported films are translated to the structure of supported films. It is, therefore, advisable to use additional techniques to analyze the pore structure of the supported materials. Gas transport measurements can be useful for this purpose [24,25].

\section{Conclusions}

- Quite similar PSDs are found for the sol-gel samples studied. A bimodal pore size distribution is found in most cases with a maximum at an effective pore diameter of around $0.5 \mathrm{~nm}$, and a weaker maximum around $0.75 \mathrm{~nm}$.

- $100 \%$ microporous non-supported silica membrane top layers have been prepared with an average porosity of $37 \%$ for samples dried under ambient conditions.

- The drying rate is found to be an important process parameter in the consolidation process. Increasing drying rates will lead to denser structures.

- Analysis of the dried and calcined non-supported membrane top layers is likely to give an overestimate of the porosity. The systems have probably, more bulk-like properties due to the long drying times compared with supported membranes.

- Incorporation of a more reactive alkoxide in the sol synthesis leads, in similar process conditions, to almost dense materials which, in some cases, contain some mesoporosity. These can be avoided if very mild reaction conditions, obtained by using less water and low reaction temperatures, are used.

- Predictions of the resulting microstructure of sols with fractal structures based on sol structure alone proved to be difficult. The reactivity of the polymers and the relative drying rate have to be incorporated.

\section{Acknowledgements}

Special thanks to J.H.A. Hekkink, A.A. ten Hoeve and P.J.A.M. Blankenvoorde for sol preparation.

\section{References}

[1] K.S.W. Sing, D.H. Everett, R.A.W. Haul, L. Moscou, R.A. Pierotti, J. Rouquérol and T. Siemieniewska, Pure Appl. Chem. 57 (1985) 603.

[2] R.J.R. Uhlhorn, K. Keizer and A.J. Burggraaf, J. Membrane Sci. 66 (1992) 271.

[3] C.J. Brinker, T.L. Ward, R. Sehgal, N.K. Raman, S.L. Hietala, D.M. Smith, D.-W. Hua and T.J. Headley, J. Membrane Sci. 77 (1993) 165.

[4] R.S.A. de Lange, J.H.A. Hekkink, K. Keizer and A.J. Burggraaf, in: Better Ceramics through Chemistry V, ed. M.J. Hampden-Smith, W.G. Klemperer and C.J. Brinker, Mater. Res. Soc. Symp. Proc., Vol. 271 (Materials Research Society, Pittsburgh, PA, 1992) p. 505. 
[5] R.S.A. de Lange, J.H.A. Hekkink, K. Keizer and A.J. Burggraaf, J. Membrane Sci. 99 (1995) 57.

[6] R.S.A. de Lange, J.H.A. Hekkink, K. Keizer and A.J. Burggraaf, J. Non-Cryst. Solids 191 (1995) 1.

[7] F.P. Cuperus, D. Bargeman and C.A. Smolders, J. Membrane Sci. 71 (1992) 57.

[8] G.Z. Cao, J. Meijerink, H.W. Brinkman and A.J. Burggraaf, J. Membrane Sci. 83 (1993) 221.

[9] S.J. Gregg and K.S.W. Sing, Adsorption, Surface Area and Porosity (Academic Press, London, 1982).

[10] G. Horváth and K. Kawazoe, J. Chem. Eng. Jpn. 16(6) (1983) 470.

[11] A. Saito and H.C. Foley, AIChE J. 37 (1991) 429.

[12] R.S.A. de Lange, K. Keizer and A.J. Burggraaf, J. Porous Mater. 1 (1995) 139.

[13] B.C. Lippens, B.G. Linsen and J.H. de Boer, J. Catal. 3 (1964) 32.

[14] B.C. Lippens and J.H. de Boer, J. Cataly. 4 (1965) 319.

[15] M.M. Dubinin, Chem. Rev. 60 (1960) 235.

[16] A. Lecloux and J.P. Pirard, J. Colloid Interf. Sci. 70 (1979) 265.

[17] C.J. Brinker and G.W. Scherer, Sol-Gel Science: The Physics and Chemistry of Sol-Gel Processing (Academic Press, San Diego, 1990) p. 558.

[18] I. Stawbridge, A.F. Craievich and P.F. James, J. Non-Cryst. Solids 72 (1985) 139.
[19] J.C. Ro and I.J. Chung, J. Non-Cryst. Solids 130 (1991) 8.

[20] A.M. Buckley and M. Greenblatt, J. Non-Cryst. Solids 143 (1992) 1.

[21] C.J. Brinker, G.C. Frye, A.J. Hurd and C.S. Ashley, Thin Solid Films 201 (1991) 97.

[22] B.B. Mandelbrot, The Fractal Geometry of Nature (Freeman, San Francisco, 1983).

[23] K.-N.P. Kumar, thesis, University of Twente, Enschede (1993).

[24] R.S.A. de Lange, J.H.A. Hekkink, K. Keizer and A.J. Burggraaf, Microporous Mater. 4 (1995) 169.

[25] R.S.A. de Lange, PhD thesis, University of Twente, Enschede (1994).

[26] R.S.A. de Lange, K. Keizer and A.J. Burggraaf, J. Membrane Sci. 104 (1994) 81.

[27] G.C. Frye, A.J. Ricco, S.J. Martin and C.J. Brinker, in: Better Ceramics Through Chemistry III, ed. C.J. Brinker, D.E. Clark and D.R. Ulrich, Mater. Res. Soc. Symp. Proc., Vol 121 (Materials Research Society, Pittsburgh, PA, 1988) p. 349 .

[28] C.J. Brinker, A.J. Hurd, P.R. Schunk and C.S. Ashley, J. Non-Cryst. Solids 147\&148 (1992) 424.

[29] B.K. Coltrain, S.M. Melpolder and J.M. Salva, in: Ultrastructure Processing of Advanced Materials, ed. D.R. Uhlmann and D.R. Ulrich (Wiley, New York, 1992) p. 69. 